\title{
UN ANÁLISIS DE LA NUCLEARIDAD PARSONIANA A PARTIR DE UNA INVESTIGACIÓN SOBRE LA RELACIÓN ENTRE ESTRUCTURA FAMILIAR Y SATISFACCIÓN PARENTAL
}

\author{
TOMASA LUENGO R. ${ }^{1}$ \\ UNIVERSIDAD DE VALLADOLID, ESPAÑA \\ (RECIBIDO EL 21/10/2008, ACEPTADO EL 11/12/2008)
}

\begin{abstract}
RESUMEN
El trabajo analiza el grado de asociación entre la satisfacción familiar de las figuras parentales y la estructura familiar de pertenencia: Familia nuclear, monoparental, binuclear o compleja. En la investigación, de naturaleza transversal, participaron 631 padres y madres de nivel sociocultural medio y medio alto. La identificación de las estructuras familiares se hizo mediante un Cuestionario de Estructuras Familiares, adaptación del Cuestionario de Familias Monoparentales (Rodríguez y Luengo, 2000, 2003) y la evaluación de la satisfacción familiar con la ESFA (Barraca y López, 1999). El estudio pone de manifiesto que los modelos familiares de corte no tradicional son menos problemáticos de lo que da a entender el funcionalismo estructural parsoniaño.
\end{abstract}

Palabras clave: Nuclear, monoparental, binuclear, satisfacción familiar.

\begin{abstract}
The study analyses of the "degree of association" between the satisfaction expressed by the parental figures and the family structure they belong to: Family nuclear, single-parent, binuclear and complex families. In the transversal investigation, 631 mothers and fathers from the middle and upper-middle socio-cultural class took part. The identification of the family structures was undertaken by means of a Family Structure Questionnaire, an adaptation of the Single-parent Family Questionnaire (Rodríguez and Luengo, 2000) and the evaluation of family satisfaction with the AFSE (Barraca and López, 1999). The study shows and that the non-traditional family models are less problematic than that explained by Parsonian structural functionalism.
\end{abstract}

Keywords: Nuclear, single-parent, binuclear, family satisfaction.

1 Docente de la Universidad de Valladolid, España. E-mail: luengo@psi.uva.es. 
"Cada uno debe abrir los ojos y no fiarse del título de parentesco ni aún de las mismas prendas de él, sino de las de amor y voluntad muy experimentados, porque no son los parientes más de cómo se trata”.

Quevedo.

\section{INTRODUCCIÓN}

Todo el siglo XX, y sobre todo la segunda mitad, fue testigo de cambios importantes en todos los campos del desarrollo humaño: cambios en la educación, trabajo, economía, relaciones humanas, familia, etc., abriendo nuevos horizontes a la experiencia humana. En el ámbito de la familia, asistimos a un reconocimiento del paradigma de la diversidad familiar desde el que se reivindica la legitimidad del pluralismo, la rápida difusión de esas tendencias sociales y el incremento de la tolerancia hacia esa diversidad.

No obstante, el reconocimiento de esas trasformaciones no ha impedido que los teóricos de la domesticidad de la institución defiendan la tesis patriarcalista de la universalidad del modelo familiar nuclear como el modelo funcional por excelencia. Así, las tesis del modelo parsoniaño, basado en la diferenciación de roles sexuales se observan en la existencia de un conjunto de preconcepciones negativas que, de alguna manera, condicionan la vida de las familias "no tradicionales". Los estudios de Fry y Addington (1984) y Rodríguez y Luengo (2000, 2003) aportan evidencia empírica de la existencia de esos estereotipos negativos sobre los hijos criados en familias monoparentales y muestran cómo la representación de la familia que comparte una sociedad, la idea del divorcio y de sus consecuencias para el desarrollo infantil, el cuerpo legal que una sociedad posee, la representación social de la responsabilidad del cuidado de la infancia, además de las actitudes ante la maternidad fuera del matrimonio, son contenidos a tener en cuenta a la hora de estudiar cualquier manifestación de la llamada "diversidad familiar", así como a la hora de diseñar y poner en práctica estrategias de intervención sobre el fenómeno.

Desde estas líneas de investigación nos proponemos aportar evidencia sobre la hipótesis de bondad de la nuclearidad parsoniana, a través de la comparación de la satisfacción familiar expresada por los progenitores de cuatro tipos de familia: (a) Familias nucleares, constituidas por los padres y madres convivientes con hijos a su cargo y cuyo proceso de formación se localiza en el matrimonio o en las nuevas formas de cohabitación; (b) familias monoparentales, constituidas por uno o varios hijos que tienen a su cabeza un solo progenitor, y cuyo proceso de formación se localiza en situaciones de desestructuración familiar y/o parentalidad biológica o social; (c) familias complejas, resultantes de la convivencia de al menos tres generaciones en sentido vertical, padres y/o madres con hijos a su cargo corresidiendo con sus progenitores de origen; y (d) familias binucleares, formadas por dos adultos y los hijos convivientes a su cargo, derivada del nuevo matrimonio o emparejamiento de los progenitores.

La familia en Parsons (1955) es un subsistema social que se articula con la personalidad de forma intrínseca y actúa en su desarrollo, por medio de la socialización, a partir de una estructura sencilla que poco a poco se vuelve más compleja. Parsons habla de dos funciones 
básicas en la familia: socialización de los hijos y estabilización de las personalidades en los adultos. Considera el proceso de socialización primaria (padres hacia hijos) como un proceso de interiorización de la cultura a partir del cual el niño puede convertirse en miembro de la sociedad en la que ha nacido. La segunda función se asocia al aspecto del creciente aislamiento de la familia nuclear de los grupos de origen de la pareja (priorización de lazos conyugales por afinidad y consanguíneos descendentes a los consanguíneos verticales ascendentes) y a la distribución de roles diferenciados y complementarios de los padres.

Para la realización de estas dos funciones, Talkott Parsons elabora un modelo basado en los roles sexuales que son mecanismos a través de los cuales las personas son integradas en el sistema social. El rol es un modelo predefinido que permite al individuo reaccionar ejerciendo funciones institucionalizadas y esperadas por su entorno social. La integración de la personalidad en el sistema social se realiza a través de la familia, que opera e interacciona condicionada por los roles establecidos.

El autor define, básicamente, cuatro roles en el interior de la familia, construidos a partir de dos ejes de diferenciación: un "eje de poder", que define roles diferentes en las diferentes generaciones (padre e hijos) y otro denominado "instrumental-expresivo" que establece la diferencia entre sexos. Cualquier sistema en el que fallaran esos roles diferenciados "estaría bajo gran tensión".

Esa atribución, que parecía haberse creado sobre bases empíricas justificables a partir de los trabajos que Bales y Slates realizaron con pequeños grupos experimentales en la década de los cincuenta, a partir de los noventa se halla fuertemente contestada en las recientes perspectivas de estudios de género, masculinidad y cuestionamientos de los modelos de sexualidad heterosexual hegemónicos. Sobres estas transformaciones en las relaciones de género intra o extrafamiliar, así como sobre los efectos sobre el bienestar de los progenitores, los trabajos de Haraway (1991), Acok y Demo, (1994); Giddens (1995), Friedman (1995), Vale de Almeida (1995) Bullok, (2001); o Demnerstein et al., (2002), son un claro exponente de la nueva corriente de pensamiento.

Para que la socialización en el interior de la familia sea estable, según la perspectiva funcionalista, sería necesario la coalición entre los roles diferenciados de padre y madre. Todo modelo distinto a este, considerado patrón ideal, es entendido como una desviación, una patología a eliminar. De esta forma, el modelo de "familia nuclear aislada" se percibe encorsetada en función de cierta rigidez al no tener en cuenta las transformaciones profundas que ya estaba sufriendo la familia en la década del cincuenta. Por ejemplo, el proceso de socialización descrito por Parsons siempre en sentido único, donde el adulto es quien socializa al niño, ignorando que en los períodos de transformación rápida o en casos de inmigración, los adolescentes y jóvenes pueden convertirse en los principales agentes de socialización de sus padres. De igual forma, fenómenos como la mayor incorporación de la mujer en el mercado de trabajo, con el crecimiento de las tasas de divorcio y separación, no han entrado dinámicamente en su modelo familiar. Y, en consecuencia, Parsons tampoco pensó que podrían acabar por provocar nuevas redefiniciones de roles en el interior de la familia como algunos autores consideran que ya está pasando. 


\section{MÉTODO}

El estudio se planteó como una investigación transversal o del momento, que toma el hogar como unidad principal de análisis y estudia la expresión de satisfacción de los padres y las madres interpretándola como cristalizaciones de historias personales.

\section{Participantes}

La muestra, de naturaleza no probabilística debido a que no existe una base de sondeo, es decir, "un documento escrito o informatizado en el que estén consignados todos y cada uno de los elementos del universo" (Ruiz-Olabuénaga et. al., 1998: 87) estuvo formada por un total de 631 progenitores $(48,7 \%$ hombres y $51,3 \%$ mujeres) entre 20 a 65 años divididos según tipo de familia en cuatro grupos (nuclear, monoparental, complejo y binuclear), los que a su vez se subdividieron en dos categorías, progenitor hombre y progenitor mujer (tabla N. ${ }^{\circ}$ ).

Tabla N. ${ }^{0}$ 1. Distribución de la muestra según tipo de familia y sexo del progenitor.

\begin{tabular}{lcccccc}
\hline & \multicolumn{2}{c}{ Hombre } & \multicolumn{2}{c}{ Mujer } & \multicolumn{2}{c}{ Total } \\
\cline { 2 - 7 } & $\mathbf{n}$ & $\mathbf{\%}$ & $\mathbf{n}$ & $\mathbf{\%}$ & $\mathbf{n}$ & $\%$ \\
\hline Nuclear & 178 & 55,6 & 49 & 15,8 & 227 & 35,8 \\
Monoparental & 24 & 7,5 & 133 & 42,8 & 157 & 24,9 \\
Compleja & 3 & 1,0 & 77 & 24,7 & 80 & 12,7 \\
Binuclear & 115 & 35,9 & 52 & 16,7 & 167 & 26,6 \\
Total & 320 & 100,0 & 311 & 100,0 & 631 & 100,0 \\
\hline
\end{tabular}

\section{Instrumentos}

Cuestionario de Estructuras Familiares. (CEF). Se trata de un cuestionario estructurado que recoge quince preguntas organizadas en tres bloques temáticos: Estructura familiar, procesos familiares y sistemas de apoyo. Su elaboración, a partir del esquema básico del Cuestionario de Familias Monoparentales de Rodríguez y Luengo (2000), fue el resultado de dos procesos paralelos: la delimitación de los objetivos generales de la investigación, su especificación y la anticipación del plan de análisis, surgiendo de todo ello la relación detallada de las dimensiones o componentes de los mismos y los indicadores de medida (Tabla N. ${ }^{\circ}$ 2). Paralelamente se recurrió a la opinión de expertos/as que ayudaron a especificar temas, recoger cuestiones, precisar terminología e, incluso, replantear preguntas. 
Tabla N. ${ }^{\circ}$ 2. Proceso de cuantificación del concepto de familia seguido en la elaboración del Cuestionario.

\begin{tabular}{cll}
\hline \multicolumn{1}{c}{ Objetivo } & \multicolumn{1}{c}{ Dimensión } & \multicolumn{1}{c}{ Indicador } \\
\hline & & A.1. Nupcialidad \\
& A. Procesos constitutivos & $\begin{array}{l}\text { A.2. Desestructuración familiar } \\
\text { A.3. Parentalidad } \\
\text { A.4. Cohabitación }\end{array}$ \\
& & \\
& & B.1. Conyugalidad \\
Concepto de familia & B. PARENTESCO (lazos legales) & B.2. Filiación \\
& & B.3. Parentalidad \\
& & B.4. Cohabitación \\
& & \\
& C. Sistemas de apoyo & C.1. Instrumentales \\
& & C.2. Afectivos \\
\hline
\end{tabular}

Cada pregunta del cuestionario consta de dos partes en su enunciado: a) el encabezamiento, que recoge el propósito de la pregunta que se formula y b) las alternativas de respuesta. Las alternativas de respuesta tipifican las preguntas en las siguientes categorías: (a) Preguntas dicotómicas, con una sola alternativa de respuesta, es la utilizada para la variable sexo; (b) Preguntas de elección múltiple, en las que se presentan tres o más posibles alternativas de respuestas. Esta formulación fue utilizada para las variables sociodemográficas del progenitor así como las relativas a la estructura familiar; (c) Diferencial Semántico en el que se presenta una escala con conceptos opuestos y se pide al progenitor que seleccione el punto que corresponde a su opinión

Escala de Satisfacción Familiar por Adjetivos. (ESFA). La Escala de Barraca y López (1999) tiene como finalidad obtener una medida objetiva de la percepción global que un sujeto tiene de su situación familiar. Está compuesta de 27 ítemes y se define por una sola dimensión por lo que se extrae una única puntuación. Su ámbito es el clínico y el investigador; es aplicable a todos los miembros de la familia a partir de los 16 años y sirve para conocer la satisfacción con la familia de origen o con la actual. El tiempo requerido para su contestación es de unos 10 minutos. La Escala, validada estadísticamente, presenta índices de fiabilidad significativamente altos.

\section{Procedimiento}

En la investigación se conjugaron dos estrategias, una estrategia cualitativa que sentó las bases conceptuales y operativas de la investigación; una segunda investigación de tipo cuantitativo que nos informó de las características de los tipos de familia estudiados y de la satisfacción familiar de los progenitores entrevistados.

La primera fase, de naturaleza exploratoria, tuvo como objetivo alcanzar una sólida orientación en el campo que se iba a investigar. Las fuentes documentales y los instrumentos que orientaron las elaboraciones conceptuales fueron: documentación descriptiva, 
documentación explicativa y entrevistas semiestructuradas a una muestra de informantes estratégicos. El resultado de todo ello fue la elaboración de los cuatro tipos de familia y del sistema de de hipótesis que someteríamos a prueba. Así, desde el paradigma de la diversidad familiar, los tipos de familia se definieron en los siguientes términos:

a) Familia nuclear: Familia constituida por los padres y madres convivientes con hijos a su cargo y cuyo proceso de formación se localiza en el matrimonio o en las nuevas formas de cohabitación.

b) Familia monoparental: Familia constituida por uno o varios hijos que tienen a su cabeza un solo progenitor, y cuyo proceso de formación se localiza en situaciones de desestructuración familiar y/o parentalidad biológica o social.

c) Familia compleja: Familia resultante de la convivencia de al menos tres generaciones en sentido vertical, padres y/o madres con hijos a su cargo corresidiendo con sus progenitores de origen.

d) Familia binuclear: Familia constituida por dos adultos y los hijos convivientes a su cargo, derivada del nuevo matrimonio o emparejamiento de los progenitores.

En cuanto al sistema de hipótesis, el modelo del estructural funcionalismo (Parsons, 1955), orientó la hipótesis principal en los siguientes términos:

Las familias "nucleares", aquellas en las que los dos padres mantienen su primer matrimonio y viven junto a sus hijos, generarán más satisfacción que: (a) las "monoparentales", por separaciones, fallecimientos o por maternidad; (b) las "complejas", derivadas de la estrategia de apoyo entre sus miembros adultos; o (d) las "reconstituidas", por el nuevo emparejamiento de uno de los cónyuges.

La segunda fase, de naturaleza cuantitativa tuvo dos objetivos. El primero, cuantificar las variables que definen el fenómeno de la diversidad familiar, a saber: a) cuantificación de las familias; b) características socioeconómicas del cabeza de familia: edad, estado civil, nivel de instrucción, situación laboral, nivel de ingresos, etc; tipo de hábitat; c) tipo de familia; d) características estructurales de la familia: número de hijos/as, edades, tamaño de la familia, etc; y e) características del sistema familiar: relación con los hijos e hijas, flexibilidad del sistema familiar, relación con el progenitor no residencial. El segundo objetivo consistió en evaluar la satisfacción familiar de los progenitores entrevistados y, con ello, estimar los niveles de satisfacción familiar expresada según tipo de familia.

Las variables cuantitativas recogidas se analizaron mediante estadísticos descriptivos de tendencia central y de dispersión y mediante un modelo lineal para la satisfacción en función de los factores tipo de familia y sexo de los progenitores. El nivel de riesgo o probabilidad de error (p) asumido para todos los contrastes de hipótesis fue del 0,05.

\section{RESULTADOS}

Los primeros datos, de naturaleza descriptiva, nos permitieron observar que la relación entre la satisfacción familiar expresada por los progenitores y el tipo de familia es significativa. Así, aunque la mayoría de los progenitores, con independencia del tipo de familia al que 
representen, valoran su satisfacción familiar como media, es de relevancia que el 31,25\% de los progenitores de los hogares complejos informa de una menor satisfacción que los padres y madres del resto de los hogares y que el 53,51\% de los progenitores de las familias monoparentales y el 33,13\% de las reconstituidas declaren mayores niveles de satisfacción que los padres y madres de los hogares nucleares $(20,70 \%)$ (Tabla N. ${ }^{\circ} 3$ ).

Tabla N. ${ }^{\circ}$ 3. Nivel de satisfacción familiar de los progenitores según tipo de familia (ambos sexos).

\begin{tabular}{lcccccccc}
\hline & \multicolumn{8}{c}{ Tipo de familia } \\
\cline { 2 - 9 } & \multicolumn{2}{c}{ Nuclear } & \multicolumn{1}{c}{ Monoparental } & \multicolumn{2}{c}{ Compleja } & \multicolumn{2}{c}{ Binuclear } \\
\cline { 2 - 10 } & $\mathbf{n}$ & $\mathbf{\%}$ & $\mathbf{n}$ & $\mathbf{\%}$ & $\mathbf{n}$ & $\mathbf{\%}$ & $\mathbf{n}$ & $\mathbf{\%}$ \\
\hline Muy baja & 27 & 11,9 & 2 & 1,3 & & & 4 & 2,4 \\
Baja & 38 & 16,7 & 4 & 2,5 & 25 & 31,3 & 6 & 3,6 \\
Normal & 115 & 50,7 & 67 & 42,7 & 42 & 52,5 & 100 & 59,9 \\
Alta & 24 & 10,6 & 24 & 15,3 & 6 & 7,5 & 31 & 18,6 \\
Muy alta & 23 & 10,1 & 60 & 38,2 & 7 & 8,7 & 26 & 15,5 \\
Total & $\mathbf{2 2 7}$ & $\mathbf{1 0 0 , 0}$ & $\mathbf{1 5 7}$ & $\mathbf{1 0 0 , 0}$ & $\mathbf{8 0}$ & $\mathbf{1 0 0 , 0}$ & $\mathbf{1 6 7}$ & $\mathbf{1 0 0 , 0}$ \\
\hline
\end{tabular}

A fin de comprobar la validez estadística de esa relación, se elaboró un modelo lineal para la satisfacción en función de los factores tipo de familia y sexo de los progenitores. Para mayor claridad presentamos los resultados completos del procedimiento estadístico seguido: ajuste del modelo, estimación de parámetros, resultados y validación del modelo.

\section{Ajuste de un modelo AñoVA de dos factores con interacción}

Variable dependiente: Satisfacción familiar, medida a través de la suma de las puntuaciones directas de la ESFA. Se ha convenido mejor usar las puntuaciones directas que los percentiles para determinar el modelo, pues de la suma de las puntuaciones se pueden obtener los percentiles correspondientes.

Factores: a) Sexo, con dos niveles: hombre o mujer y b) tipo de familia, con cuatro niveles: nuclear, monoparental, compleja, binuclear.

El modelo responde a la siguiente ecuación:

$$
\text { Satisfacción }_{\mathrm{ij}}=\alpha+\beta * \operatorname{sexo}_{\mathrm{i}}+\gamma \square * \text { familia }_{\mathrm{j}}+\delta * \operatorname{sexo}+\text { familia }_{\mathrm{ij}}+\varepsilon_{\mathrm{ij}}
$$

Donde: $\mathrm{y}_{\mathrm{ij}}$ : Puntuación de un individuo con nivel i en el factor sexo y nivel j del factor tipo de familia; $\alpha$ : Término independiente o constante del modelo; sexo: Sexo del individuo: hombre o mujer; familia: Tipo de familia a la que pertenece el individuo: nuclear, monoparental, compleja, binuclear. 
El análisis de los resultados recogidos en la tabla N. ${ }^{\circ} 4$ permite observar que a) El modelo explica un 27,3\% de la variabilidad total de la satisfacción familiar, como puede apreciarse en el $\mathrm{R}^{2}$ corregido (.273). b) Se rechaza la hipótesis nula de falta de relación entre la variable dependiente y los factores $(\mathrm{p} \leq 05)$. c) Los factores sexo $(\mathrm{F}=5,6 ; \mathrm{p}$ $\leq .019)$ y tipo de familia $(\mathrm{F}=7,2 ; \mathrm{p} \leq .000)$ tienen un efecto muy significativo en la satisfacción del individuo. El efecto de la interacción $(F=6,4 ; p \leq .000)$ entre ambos factores también es muy significativo, por lo que el efecto que el tipo de familia produce sobre la satisfacción es dependiente del efecto del sexo y viceversa.

Tabla N. ${ }^{0}$ 4. Tabla AñoVA de ajuste del modelo.

\begin{tabular}{lccccc}
\hline \multicolumn{6}{c}{ Variable dependiente: suma de puntuaciones directas de la ESFA } \\
\hline \multicolumn{1}{c}{ Fuente } & $\begin{array}{c}\text { Suma de cuadrados } \\
\text { tipo III }\end{array}$ & gl & $\begin{array}{c}\text { Media } \\
\text { cuadrática }\end{array}$ & F & Significación \\
\hline Modelo corregido & 23094,7 & 7 & 3299,2 & 34,8 & 0,000 \\
Intersección & 2389153,6 & 1 & 2389153,6 & 25208,9 & 0,000 \\
Sexo & 527,3 & 1 & 527,3 & 5,6 & 0,019 \\
Tipo de familia & 2063,6 & 3 & 687,9 & 7,2 & 0,000 \\
Sexo x tipo familia & 1814,1 & 3 & 604,7 & 6,4 & 0,000 \\
Error & 59044,3 & 623 & 94,8 & & \\
Total & 10421211,0 & 631 & & & \\
Total corregida & 82138,9 & 630 & & & \\
R cuadrado $=.281$ (R cuadrado corregida $=.273)$ & & & \\
\hline
\end{tabular}

Estimaciones de los parámetros. Para establecer el modelo definitivo, se estimaron los parámetros que aparecen en la ecuación del mismo. En este caso, como se ha utilizado el programa estadístico SPSS, en la salida del programa aparecen, no las estimaciones de los parámetros, sino las estimaciones de los efectos que producen los factores, lo que se recoge en la tabla $\mathrm{N}^{\circ}{ }^{\circ}$. Por efecto se entiende el incremento o decremento que se produce en la media general ( $\alpha$ o intersección).

Mediante el modelo ajustado, se estimó las puntuaciones directas de un individuo sumando los efectos correspondientes: Ejemplo: Para un hombre nuclear: satisfacción $=$ Intersección $+[$ Sexo $=$ Hombre $]+[$ Tipo $=$ Nuclear $]+[$ Sexo $=$ Hombre $] *[$ Tipo $=$ Nuclear $]=$ $134,2-5,7-1,6-6,1=120,8$ (Tabla N. ${ }^{\circ}$ 5).

Los resultados en relación a las estimaciones de las medias marginales de los efectos se presentan a continuación. 
Tabla 5. Estimaciones de los efectos.

\begin{tabular}{|c|c|c|c|c|c|c|}
\hline \multicolumn{7}{|c|}{ Variable dependiente: suma puntuaciones directa de la ESFA } \\
\hline \multirow[b]{2}{*}{ Parámetro } & \multirow[t]{2}{*}{ B } & \multirow[t]{2}{*}{ Error típ. } & \multirow[t]{2}{*}{$\mathbf{t}$} & \multirow[t]{2}{*}{ Significación } & \multicolumn{2}{|c|}{$\begin{array}{c}\text { Intervalo de } \\
\text { confianza al 95\% }\end{array}$} \\
\hline & & & & & $\begin{array}{l}\text { Límite } \\
\text { inf. }\end{array}$ & $\begin{array}{l}\text { Límite } \\
\text { sup. }\end{array}$ \\
\hline Intersección & 134,2 & 1,3 & 99,4 & 0,0 & 131,6 & 136,9 \\
\hline$[$ Sexo $=$ Hombre $]$ & $-5,7$ & 1,6 & $-3,5$ & 0,0 & $-8,9$ & $-2,5$ \\
\hline$[$ Sexo $=$ Mujer $]$ & 5,7 & $-0,3$ & $-20,6$ & 0,0 & 5,2 & 6,3 \\
\hline$[$ Tipo $=$ Nuclear $]$ & $-1,6$ & 1,9 & $-0,8$ & 0,4 & $-5,4$ & 2,2 \\
\hline$[$ Tipo $=$ Monoparental $]$ & 1,8 & 1,6 & 1,1 & 0,2 & $-1,3$ & 4,9 \\
\hline$[$ Tipo $=$ Compleja $]$ & $-11,3$ & 1,7 & $-6,5$ & 0,0 & $-14,7$ & $-7,9$ \\
\hline$[$ Tipo $=$ Binuclear $]$ & 12,1 & $-3,9$ & $-3,1$ & 0,0 & 18,8 & 3,4 \\
\hline$[$ Sexo $=$ Hombre $] *[$ Tipo $=$ Nuclear $]$ & $-6,1$ & 2,3 & $-2,7$ & 0,0 & $-10,6$ & $-1,7$ \\
\hline$[$ Sexo $=$ Hombre $] *[$ Tipo $=$ Monoparental $]$ & $-3,2$ & 2,7 & $-1,2$ & 0,2 & $-8,5$ & 2,1 \\
\hline$[$ Sexo $=$ Hombre $] *[$ Tipo $=$ Compleja $]$ & 16,8 & 5,9 & 2,8 & 0,0 & 5,1 & 28,5 \\
\hline$[$ Sexo $=$ Hombre $] *[$ Tipo $=$ Binuclear $]$ & $-13,2$ & $-9,3$ & 1,4 & 0,0 & $-31,4$ & 5,0 \\
\hline$[$ Sexo $=$ Mujer $] *[$ Tipo $=$ Nuclear $]$ & 4,5 & $-0,3$ & $-14,0$ & 0,0 & 5,1 & 3,9 \\
\hline$[$ Sexo $=$ Mujer $] *[$ Tipo $=$ Monoparental $]$ & 5,0 & $-1,1$ & $-15,1$ & 0,0 & 2,8 & 8,2 \\
\hline$[$ Sexo $=$ Mujer $] *[$ Tipo $=$ Compleja $]$ & $-28,1$ & $-4,2$ & 6,7 & 0,0 & $-36,4$ & $-19,9$ \\
\hline$[$ Sexo $=$ Mujer $] *[$ Tipo $=$ Binuclear $]$ & 24,3 & 5,4 & 4,5 & 0,0 & 13,7 & 34,8 \\
\hline
\end{tabular}

Nota: Aquellos efectos que son significativos se han resaltado en negrita.

Tabla N. ${ }^{\circ}$ 6. Puntuaciones predichas de la satisfacción para un individuo.

\begin{tabular}{lcccc}
\hline \multirow{2}{*}{ Sexo } & \multicolumn{4}{c}{ Tipo de familia } \\
\cline { 2 - 5 } & Nuclear & Monoparental & Compleja & Binuclear \\
\hline Hombre & 120,8 & 127,1 & 134,0 & 128,5 \\
Mujer & 132,6 & 136,0 & 122,9 & 134,2 \\
\hline
\end{tabular}

Resultados para la variable Sexo:

Tabla N. ${ }^{0}$ 7. Medias marginales estimadas por sexo.

\begin{tabular}{|c|c|c|c|c|}
\hline \multicolumn{5}{|c|}{ Variable dependiente: suma de puntuaciones directas de la ESFA } \\
\hline \multirow{2}{*}{ Sexo } & \multirow[t]{2}{*}{ Media } & \multirow[t]{2}{*}{ Error típ. } & \multicolumn{2}{|c|}{ Intervalo de confianza al $\mathbf{9 5 \%}$} \\
\hline & & & Límite inferior & Límite superior \\
\hline Hombre & 127,6 & 1,5 & 124,6 & 130,6 \\
\hline Mujer & 131,4 & 0,6 & 130,3 & 132,6 \\
\hline
\end{tabular}


Tabla N. ${ }^{0}$ 8. Comparaciones por pares para la variable sexo.

\begin{tabular}{|c|c|c|c|c|c|c|}
\hline \multicolumn{7}{|c|}{ Variable dependiente: suma de puntuaciones directas de la ESFA } \\
\hline \multirow{2}{*}{ (I) Sexo } & \multirow{2}{*}{ (J) Sexo } & \multirow[t]{2}{*}{$\begin{array}{l}\text { Diferencia entre } \\
\text { medias (I-J) }\end{array}$} & \multirow[t]{2}{*}{$\begin{array}{l}\text { Error } \\
\text { típ. }\end{array}$} & \multirow[t]{2}{*}{ Significación } & \multicolumn{2}{|c|}{$\begin{array}{l}\text { Intervalo de confianza al } \\
95 \% \text { para diferencia }\end{array}$} \\
\hline & & & & & $\begin{array}{c}\text { Límite } \\
\text { inferior }\end{array}$ & $\begin{array}{c}\text { Límite } \\
\text { superior }\end{array}$ \\
\hline Hombre & Mujer & $-3,8$ & 1,6 & 0,0 & $-7,0$ & $-0,6$ \\
\hline Mujer & Hombre & 3,8 & 1,6 & 0,0 & 0,6 & 7,0 \\
\hline * & \multicolumn{6}{|c|}{ La diferencia de las medias es significativa al nivel, 05 . } \\
\hline a & \multicolumn{6}{|c|}{ Ajuste para comparaciones múltiples: Diferencia menos significativa } \\
\hline
\end{tabular}

Figura $\mathbf{N}^{0}$ 1. Comparaciones por pares para la variable sexo.

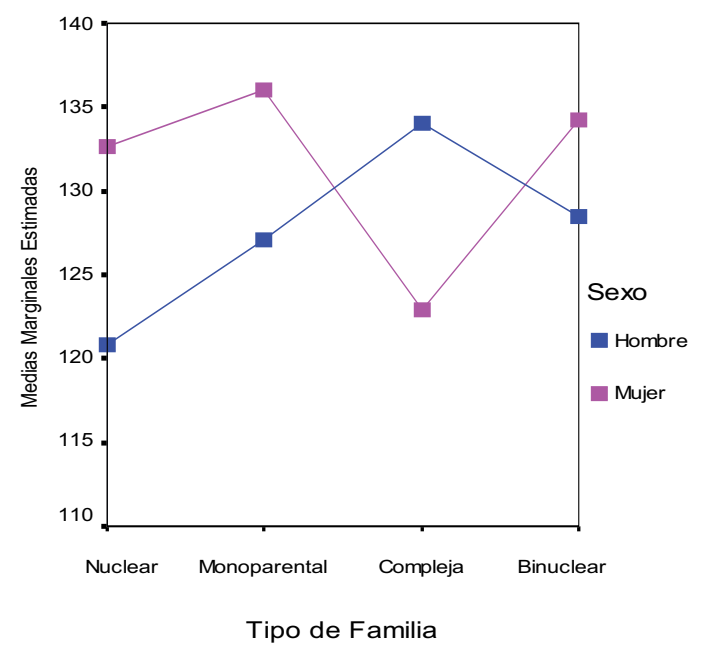

Como puede observarse en la tabla N. ${ }^{\circ} 7$ y figura 1 , los datos del test de diferencia de medias para los niveles del factor sexo indican que la satisfacción media para los hombres y las mujeres es distinta y, en concreto, es significativamente mayor la de las mujeres. Estos datos no se confirman para las madres de las familias complejas cuya puntuación 122,9 es casi 11 puntos menor respecto a la de los progenitores varones $(134,0)$. No obstante, estas diferencias pueden no ser significativas, dada la no significación del tamaño muestral de los progenitores varones de familias complejas.

\section{Resultados para la variable Tipo de Familia:}

En la tabla de contraste 9 y figura N. ${ }^{\circ} 2$ se muestra cómo la satisfacción de los progenitores residentes en familias nucleares es significativamente menor que la satisfacción de padres y madres de los tipos monoparental y binuclear. Para el resto de tipos de familias no existen diferencias significativas en cuanto a la satisfacción. 
Tabla N. ${ }^{\circ}$ 9. Medias marginales estimadas por tipo de familia-

\begin{tabular}{lccccc}
\hline & \multicolumn{3}{c}{ Variable dependiente: suma de puntuaciones directas de la ESFA } \\
\cline { 2 - 3 } Tipo Familia & Media & Error típ. & & \multicolumn{2}{c}{ Intervalo de confianza al 95\% } \\
\cline { 2 - 3 } \cline { 5 - 6 } Nuclear & 126,7 & & Límite inferior & Límite superior \\
Monoparental & 131,6 & 1,1 & & 125,2 & 128,2 \\
Compleja & 128,4 & 2,9 & & 122,8 & 133,7 \\
Binuclear & 131,4 & 0,8 & & 129,8 & 134,1 \\
\hline
\end{tabular}

Tabla N. ${ }^{\circ}$ 10. Comparaciones por pares según tipo de familia.

\begin{tabular}{|c|c|c|c|c|c|c|}
\hline \multicolumn{7}{|c|}{ Variable dependiente: suma de puntuaciones directas de la ESFA } \\
\hline \multirow{2}{*}{ (I) Tipo } & \multirow{2}{*}{ (J) Tipo } & \multirow[t]{2}{*}{$\begin{array}{c}\text { Diferencia } \\
\text { entre medias } \\
(\mathrm{I}-\mathrm{J}) \\
\end{array}$} & \multirow[t]{2}{*}{$\begin{array}{c}\text { Error } \\
\text { típ. }\end{array}$} & \multirow[t]{2}{*}{ Significación } & \multicolumn{2}{|c|}{$\begin{array}{l}\text { Intervalo de confianza } \\
\text { al 95\% para diferencia }\end{array}$} \\
\hline & & & & & $\begin{array}{c}\text { Límite } \\
\text { inferior }\end{array}$ & $\begin{array}{c}\text { Límite } \\
\text { superior }\end{array}$ \\
\hline \multirow[t]{3}{*}{ Nuclear } & Monoparental & $-4,9$ & 1,3 & 0,0 & $-7,5$ & $-2,2$ \\
\hline & Compleja & $-1,7$ & 3 & 0,5 & $-7,6$ & 4,1 \\
\hline & Binuclear & $-4,7$ & 1,1 & 0,0 & $-6,9$ & $-2,4$ \\
\hline \multirow[t]{2}{*}{ Monoparental } & Compleja & 3,1 & 3,1 & 0,3 & $-2,8$ & 9,1 \\
\hline & Binuclear & 0,2 & 1,3 & 0,9 & $-2,4$ & 2,9 \\
\hline Compleja & Binuclear & $-2,9$ & 3 & 0,3 & $-8,8$ & 2,9 \\
\hline \multicolumn{7}{|c|}{ Basadas en las medias marginales estimadas. } \\
\hline * & \multicolumn{6}{|c|}{ La diferencia de las medias es significativa al nivel .05 . } \\
\hline $\mathrm{a}$ & \multicolumn{6}{|c|}{ Ajuste para comparaciones múltiples: Diferencia menos significativa } \\
\hline
\end{tabular}

Figura N. ${ }^{\circ}$ 2. Comparaciones por pares según tipo de familia.

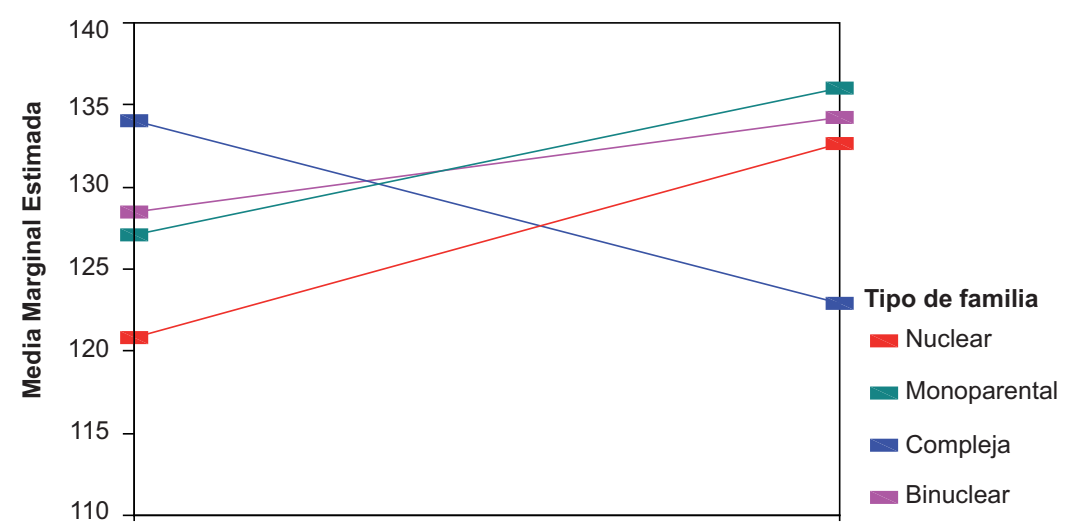


Validación del modelo. Para validar el modelo se comprobaron dos hipótesis sobre los residuos del modelo: Normalidad y Homocedasticidad. En la validación de la hipótesis de normalidad se han utilizado dos métodos, el gráfico (Plot) de Normalidad de los residuos y la prueba de Kolmogorov-Smirnov. Mediante la prueba gráfica se constata que los valores "observados" de los residuos frente a los valores "esperados" de los mismos, coinciden en gran medida (figura N. ${ }^{\circ} 3$ ) lo que permite concluir que no hay evidencias para rechazar la hipótesis de que la distribución de los residuales sea normal. Sólo se aprecian desviaciones de la normalidad en las colas de la distribución, suceso bastante habitual y que no merece ninguna importancia.

Figura N. ${ }^{\circ}$ 3. Plot de normalidad.

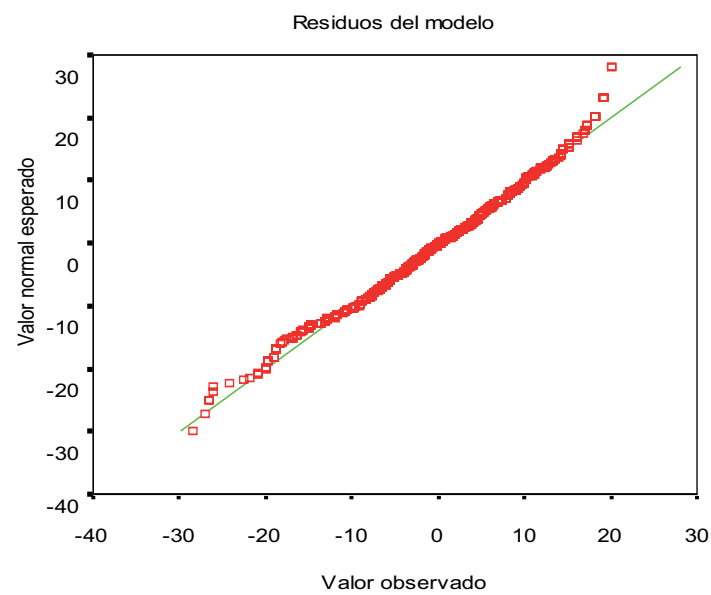

Esta conclusión es confirmada mediante la prueba de Kolmogorov-Smirnov, cuyos resultados se exponen en la tabla N. ${ }^{\circ} 11$. En efecto, a la vista del p valor $(0,4)$ (probabilidad de error del $40 \%$ ) no se puede rechazar que los residuos se distribuyan de manera normal.

Tabla N. ${ }^{0}$ 11. Prueba de Kolmogorov-Smirnov para los residuos.

\begin{tabular}{llc}
\hline \multicolumn{3}{c}{ Residuo } \\
\hline N & & 631 \\
Parámetros normales & Media & 0,0 \\
& Desviación típica & 9,7 \\
& Absoluta & 0,0 \\
Diferencias más extremas & Positiva & 0,0 \\
& Negativa & $-0,0$ \\
Z de Kolmogorov-Smirnov & & 0,9 \\
Sig. asintót. (bilateral) & & 0,4 \\
\multicolumn{2}{c}{ a } & La distribución de contraste es la Normal. \\
b & Se han calculado a partir de los datos. \\
\hline
\end{tabular}


Para validar la hipótesis de homocedestacidad de los residuos, se ha comprobado que la varianza de los residuos es más o menos constante para todas las observaciones. Para ello, se han utilizado dos pruebas: el diagrama de dispersión de los residuos y el contraste de Levene. La prueba gráfica de dispersión de los residuos (figura N. ${ }^{\circ} 4$ ) no aporta evidencia clara de si realmente los residuos tienen la misma variabilidad (homogeneidad de la varianza) a lo largo de los grupos por lo que se recurrió a un contraste analítico mediante la prueba de Levene. Como puede observarse en la tabla N. ${ }^{\circ} 12$, la prueba de Levene contrasta la hipótesis nula de que la varianza del error de la variable dependiente es igual u homogénea a lo largo de todos los grupos. En este caso se rechaza la homocedasticidad de la varianza, pero esto no lleva a rechazar el modelo, pues esta heterocedasticidad era previsible al tener uno de los grupos (hombres complejos) un tamaño muy inferior al resto de los grupos. En general cuando esto ocurre y el tamaño de los grupos que definen los factores es más o menos similar, no importa que esta hipótesis se rechace para poder validar el modelo.

Figura N. ${ }^{4}$. Diagrama de dispersión de los residuos.

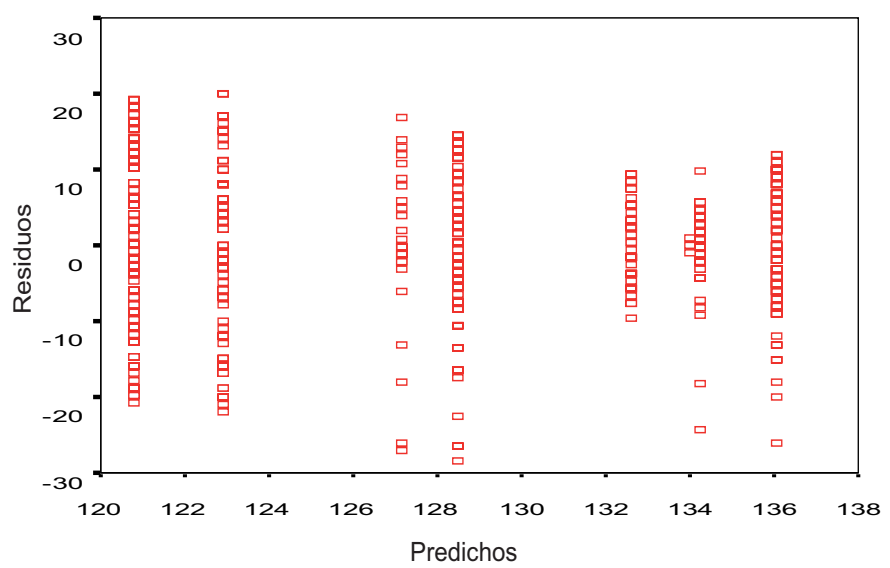

Tabla N. ${ }^{0}$ 12. Contraste de Levene sobre la igualdad de las varianzas error.

\begin{tabular}{cccc}
\hline \multicolumn{4}{c}{ Variable dependiente: } \\
\hline $\mathrm{F}$ & $\mathrm{g}$ l1 & $\mathrm{g} 12$ & Significación \\
17,66 & 7 & 623 & 0,00 \\
\hline
\end{tabular}

\section{DISCUSIÓN Y CONCLUSIONES}

Aunque en nuestra investigación hemos recurrido a los análisis estadísticos que nos permitieran hacer inferencias descriptivas y explicativas, somos concientes de que la inferencia es, por definición, un proceso imperfecto. El objetivo en nuestra investigación fue utilizar datos cuantitativos para conocer las familias que los han producido. Evidentemente es imposible llegar a conclusiones perfectamente ciertas utilizando datos inciertos. De hecho, la incertidumbre es un aspecto crucial de toda investigación o conocimiento del 
mundo. Aún así, los resultados que hemos presentados nos permiten apuntar las siguientes consideraciones:

Desde un punto de vista teórico, la muestra de nuestra investigación no ha respondido a los supuestos estructurales y funcionalistas de Parsons. A través de este estudio, hemos podido observar que las madres y los padres de las familias de corte no tradicional (familias que viven el posdivorcio, familias reconstituidas y familias de madres solteras) son mucho menos problemáticas de lo que da a entender el funcionalismo estructural tradicional. La solidaridad afectiva resulta ser mucho más importante que lo que expresa el modelo de nuclearidad parsoniaño. Las perspectivas funcionalistas estructurales no anticiparon el concepto de padres no residentes y padrastros como potenciales participantes activos dentro de la familia; es más, la participación significativa y regular de estos padres no tradicionales produce efectos sobre las interacciones familiares que el otro progenitor valora de forma positiva.

Los hallazgos de esta investigación ponen en duda supuestos y estereotipos populares vinculados a la ideología de la familia nuclear de tipo tradicional. A través de la relación ente las distintas variables y la satisfacción expresada por los progenitores, se ha constatado un modelo uniforme en el que se advierte que la discrepancia que existe dentro de cada familia es mayor que la que existe en los diferentes tipos de familia. Estos hallazgos representan un reto para los investigaciones que siguen fundándose en categorizaciones de la estructura familiar y supuestos tales como el de una familia nuclear de cónyuges en primeras nupcias (mejor si la madre no trabaja, pero, por lo menos, que sea la principal responsable del cuidado de los hijos) facilitando el mejor entorno para la felicidad conyugal y educar niños adaptables y saludables. La evidencia es convincente en cuanto a que estas variables, por sí solas, explican muy poco sobre las pautas de interacción habituales y las experiencias inmediatas que construyen la satisfacción familiar de los padres y las madres.

Por último, los datos de nuestra muestra apoyan las tesis de que las familias han sido siempre mucho más diversas de lo que sugiere la nostalgia que se asocia a la familia nuclear. El aumento de las diferentes estructuras familiares significa que la mayoría de las familias ya no se parecen a ese modelo de "familia idílica" en el que la "estructura intacta" y la "división de papeles" garantiza la socialización funcional de progenitores e hijos. Desde un punto de vista organizativo, se ha evolucionado de un modelo centro-patriarcal en el cual el padre era el jefe de la familia y la madre el polo afectivo, a un modelo centrado en el hijo, en el que la idea de jefe de la familia se diluye en la noción de autoridad parental conjunta y el polo afectivo se inclina cada vez más hacia el niño. El ejercicio de la parentalidad se está transformando y este cambio representa uno de los grandes retos para las sociedades y los profesionales al servicio del desarrollo humaño:

Acompañar al padre patriarcal en la transición hacia el padre presente, igualitario, participativo, que comparte la vida familiar. Ayudarles a ceder sus "derechos tradicionales", a renunciar a ser el importante de la familia, a conquistar ser un adulto más. 


\section{REFERENCIAS BIBLIOGRÁFICAS}

1. Acok, A.C. y Demo, D.H. (1994). Family Diversity and Well-Being. Newburry Parck: C.A. Sage.

2. Barraca, J. y López-Yarto, L (1999). Escala de Satisfacción Familiar por Adjetivos. Madrid: TEA.

3. Bullock, K. (2001). Healthy family systems? The changing role of grandparents in rural America, Education and ageing, 16. (pp. 163-178).

4. Dennerstein, Lorraine; Dudley, E.; Guthrie, J. (2002). Empty nest or revolving door? A prospective study of women's quality of life in midlife during the phase of children leaving and re-entering the home, Psychological Medicine, 3 (vol. 32) (pp. 545-550).

5. Friedman, S. (1995). Beyond White and Other: Relationality and narratives of rece in feminist discourse. En Signis, Journañ of Women in Cultur and society, vol.1, $\mathrm{n}^{\mathrm{o}}$ 1. University of Chicago.

6. Fry, P.S. y Addington J. (1984). Professionals negative expectations of boys from father-headed single-parent families: Implicationes for the training of child-care professional. British Journal of Developmental Psychology, 2, 337-346.

7. Giddens, A. (1995). La transformación de la intimidad. Madrid: Cátedra.

8. Haraway, D. (1991). Ciencia, cyborgs y mujeres. La reinvención de la naturaleza. Universidad de Valencia, Instituto de la Mujer. Madrid: Cátedra.

9. Luengo Rodríguez, T. y Román Sánchez, J.M. (2005) "La familia postmoderna: procesos constitutivos y parentalidad", en Romay Martínez, J. y García Mira, R. Psicología social y problemas sociales. Psicología ambiental, comunitaria y de la educación. Madrid. Biblioteca Nueva, 581-588.

10. Luengo Rodríguez, Tomasa (2008). “Mujer y familia. Un área para la intervención”, en Servicios sociales y política social, 81: 63-80. Número dedicado a viejas y nuevas pobrezas. Consejo General de Colegios Oficiales de Diplomados en Trabajo Social. Madrid.

11. Nerirand, G. (2001). Mutations sociales et reversement des perspectives sur la parentalité. En D. Le Gall et Y. Bettahar, La pluriparentaleté. Paris: PUF.

12. Parsons, T.y Bales, R. (comps.), (1955). Family, socialization and interaction process. Nueva York: Free Press.

13. Rodríguez, C. y Luengo, T. (2003). Un análisis del concepto de familia monoparental a partir de una investigación sobre núcleos familiares monoparentales. Papers, 69 (pp. 59-82).

14. Rodríguez, C. y Luengo, T. (dirs.), (2000). Las familias monoparentales en Castilla y León. Valladolid: Junta de Castilla y León, Consejería de Sanidad y Bienestar Social.

15. Ruiz, Olabuénaga, J.I.; Aristegui, I. y Melgosa, L. (1998). Cómo elaborar un proyecto de investigación social. Bilbao: ICE, Universidad de Deusto.

16. Vale De Almeida, M. (1995). Senhores de si: uma interpretaçao antropológica da masculinidade. Lisboa, Fim de século. 